\title{
Immune cells transferred by colostrum do not influence the immune responses to foot-and-mouth disease primary vaccination
}

\author{
Danilo Bucafusco, ${ }^{1,2}$ Rodrigo Pereyra, ${ }^{3}$ Florencia C. Mansilla, ${ }^{1,2}$ Darío A. Malacari, ${ }^{1}$ María S. Juncos, ${ }^{4}$ \\ Sebastián Di Giacomo, ${ }^{1,2}$ Andrea F. Ayude, ${ }^{1,2}$ Mariano Pérez-Filgueira, ${ }^{1,2}$ and Alejandra V. Capozzo ${ }^{1,2 *}$ \\ ${ }^{1}$ Instituto Nacional de Tecnología Agropecuaria, Centro de Investigaciones en Ciencias Veterinarias y Agronómicas, Instituto de Virología, \\ Hurlingham, Buenos Aires, Argentina 1686 \\ ${ }^{2}$ Consejo Nacional de Investigaciones Científicas y Técnicas (CONICET), Instituto de Virología e Innovaciones Tecnólogicas (IVIT), \\ Hurlingham, Buenos Aires, Argentina 1686 \\ ${ }^{3}$ Instituto Nacional de Tecnología Agropecuaria, Estación Experimental Cerillos, Salta, Argentina 4403 \\ ${ }^{4}$ Independent Veterinarian, Tandil, Buenos Aires, Argentina 7000
}

\section{ABSTRACT}

Little is known about the influence of maternal antibodies and immune cells transferred through colostrum on the immune responses of calves to the currently used foot-and-mouth disease (FMD) vaccines. Here we evaluated the humoral and cellular immune responses induced by vaccination of colostrum-deprived calves and calves that received equivalent amounts of colostrum preparations that differed in the presence or absence of maternal immune cells but contained the same quantity and quality of anti-foot-and-mouth disease virus (FMDV) antibodies. Three groups of 32-d-old calves ( $\mathrm{n}=3$ per group) were deprived of colostrum and fed either whole immune colostrum or a cell-free colostrum preparation containing only anti-FMDV antibodies. All groups were immunized with 1 dose of an oil-adjuvanted commercial vaccine. Blood samples were collected periodically before vaccination and weekly after vaccination. Immune responses specific to FMDV were assessed based on T-cell proliferation, IFN- $\gamma$ production, total and neutralizing serum antibodies, and isotype profile. All vaccinated calves developed IFN- $\gamma$ and lymphoproliferative responses, irrespective of the colostrum received. Colostrum-deprived animals responded to vaccination with a primary $\operatorname{IgM}$ response followed by an increase of $\operatorname{IgG}_{1}$ titers. Conversely, antibody titers decreased in all colostrum-fed calves after vaccination. This study demonstrates for the first time that maternal immune cells transferred to the calves through colostrum do not modify immune responses to FMD vaccine, and it confirms the interference of maternal antibodies in the induction of humoral but not cell-mediated immune responses.

Received October 1, 2018.

Accepted February 13, 2019.

*Corresponding author: capozzo.alejandra@inta.gob.ar
Key words: foot-and-mouth disease vaccine, maternal immunity, calves, colostrum

\section{INTRODUCTION}

Colostrum contains high concentrations of nutrients and hormones to support growth and maturation in the physiological development of the calf (Goldman, 1993). Bovine colostrum also contains maternal antibodies (MAB) and a large number of maternal immune cells (Mohr et al., 1970; Reber et al., 2008). Several studies have addressed the role of antibodies passively transferred by colostrum in neonatal protection (Gay et al., 1965; Belknap et al., 1991) and how these antibodies interact with incoming pathogens or with vaccination (Ridpath et al., 2003; Bucafusco et al., 2014). However, the influence of the maternal leukocytes in the development of the calves' immune responses to pathogens or vaccines still needs to be defined. Donovan et al. (2007) reported that transfer of live maternal cells from colostrum to neonatal calves enhanced responses to antigens against which the dams had previously been exposed, but not to antigens to which the dams were naïve. Other studies demonstrated that calves fed with whole colostrum have enhanced expression of activation markers and major histocompatibility complex II (Reber et al., 2008; Langel et al., 2015). Previous studies also showed that adoptive transfer of maternal cells through colostrum affects the development of the neonatal immune system (Langel et al., 2016). These studies suggest that calves fed whole colostrum are able to activate immune cells, leading to enhanced responses upon antigen exposure.

Vaccines based on inactivated viral antigens are usually capable of inducing T-cell responses but not humoral responses in competent young individuals in the presence of MAB (Endsley et al., 2003; Vitour et al., 2011). We previously demonstrated that the induc- 
tion of anti-foot-and-mouth disease virus (FMDV) neutralizing antibodies and IgM-driven primary responses to vaccination only became evident as MAB titers decreased (Bucafusco et al., 2014). Current footand-mouth disease (FMD) vaccines in Argentina are oil-adjuvanted and contain 4 binary ethylenimine-inactivated FMDV strains of the latest regional circulation: O1/Campos/Brazil/58 (O1/Campos), A24 Cruzeiro/ Brazil/55 (A24/Cruzeiro), A/Argentina/2001 (A/ Arg/2001), and C3/Indaial/Brazil/71 (C3/Indaial) (SENASA, 2006, 2013). Animals older than $2 \mathrm{yr}$ are vaccinated once a year, while immunizations are performed every 6 mo in calves up to 2 yr old (SENASA, 2013). Consequently, calves may be vaccinated when they still have maternally derived immunity transferred through colostrum.

The aim of this study was to assess the magnitude and kinetics of the humoral and cellular immunity induced by primary FMD vaccination in calves fed with colostrum preparations that differed only in the presence or absence of maternal cells. We measured total and neutralizing antibodies in the vaccinated calves, characterized the isotypes induced, and evaluated cellmediated immunity in terms of lymphoproliferation and production of systemic IFN- $\gamma$. This study provides new insights on the immunogenicity of current FMD oil-adjuvanted vaccines used in the presence of maternal immunity.

\section{MATERIALS AND METHODS}

\section{Animals and Experimental Design}

This study used crossbred Jersey/Holstein calves that were born to multivaccinated cows or heifers (last vaccination $\sim 5$ mo before calving) raised on a dairy farm located in Tandil, Buenos Aires, Argentina. Animals were monitored to prevent colostrum intake from the moment of birth. Once a newborn calf was able to stand up, it was moved to a separate room for perinatal care. After a general objective exam, the residual amniotic fluid was removed and the umbilical cord was disinfected with iodinated alcohol.

Calves were divided into 3 experimental groups $(\mathrm{n}=3$ each): deprived of colostrum (CD), receiving whole colostrum (WC), or receiving acellular colostrum (AC). Animals born to cows were randomly assigned to the WC or AC group. Calves born to heifers were assigned to the CD group and received a standard treatment according to protocols developed by our group (Malacari, 2016; Malacari et al., 2018). An additional group of 5 animals were fed $2 \mathrm{~L}$ of the whole colostrum preparation (as described below) and left unvaccinated. These animals were bled at $0,21,35,49$, and $60 \mathrm{~d}$ after birth, and serum samples were analyzed by various serological methods (see below) to estimate the half-life of MAB.

Calves were kept at the farm for the first $24 \mathrm{~h}$ of life before being transferred to biosafety level 2 animal boxes at Instituto Nacional de Tecnología Agropecuaria (INTA), where they remained throughout the experiment. The WC and AC calves were fed $2 \mathrm{~L}$ of colostrum within the first $6 \mathrm{~h}$ of life and then received another $2 \mathrm{~L}$ before $12 \mathrm{~h}$ of life. The CD calves were fed a $2 \times$ concentrated milk replacer AF 80 (ACA, San Nicolás, Buenos Aires, Argentina), following the same volumes and schedule as colostrum-fed animals. In all cases, an esophageal tubing procedure was employed to ensure correct and uniform administration. Blood samples (10 $\mathrm{mL}$ ) were collected from the jugular vein of calves at 6 to $12 \mathrm{~h}$ after the last administration of colostrum to corroborate the transfer of MAB. Blood samples were again collected at 1, 15, and $32 \mathrm{~d}$ of age and at 7, 14, 21, and $28 \mathrm{~d}$ post-vaccination (DPV). Samples were taken using Vacutainer (BD, Franklin Lakes, NJ) tubes for serum separation or tubes containing sodium heparin (samples for analyzing cellular immune responses).

\section{Colostrum Preparations}

Acellular colostrum preparations (cell-free colostrum) were produced from approximately $30 \mathrm{~L}$ of colostrum collected at the same dairy farm from cows that had calved the same day. Colostrum was cooled to $4^{\circ} \mathrm{C}$ and transported to our laboratory where it was stored at $-20^{\circ} \mathrm{C}$ for $48 \mathrm{~h}$. To pellet remaining cells and cell debris, colostrum was thawed in a heat bath at $37^{\circ} \mathrm{C}$ and centrifuged in $350-\mathrm{mL}$ fractions at 3,000 $\times g$ for $20 \mathrm{~min}$ in $500-\mathrm{mL}$ bottles. The fat fraction of each flask was recovered and placed together with the aqueous fraction in a beaker, and the 2 phases were homogenized. The cell fractions were discarded. Colostrum resulting from this process was aliquotted into 2 -L bottles and frozen at $-20^{\circ} \mathrm{C}$. Upon the birth of a calf, a bottle was thawed by immersion in cold water and then warmed to $37^{\circ} \mathrm{C}$ to be administered as described above.

Four liters of whole colostrum was obtained by hand milking. Each calf was fed with colostrum from its own dam to ensure the viability of the immune cells by minimizing handling. They were fed according to the procedure described above.

\section{Vaccine}

We used an officially approved commercial tetravalent single-oil emulsion vaccine containing inactivated FMDV strains A24/Cruzeiro, A/Arg/2001, O1/Cam- 
pos, and C3/Indaial. The vaccine was injected subcutaneously in the neck (2 mL/dose) according to the manufacturer's recommendations and official regulations (SENASA, 2006).

\section{Serological Assays}

Serology included a virus neutralization test (VNT), liquid phase blocking ELISA (LPBE), and isotype ELISA against the O1/Campos strain. All serological tests were performed according to previously published reports, following standard protocols developed or modified in our laboratory (Lavoria et al., 2012; Brito et al., 2014; Bucafusco et al., 2014).

Serum neutralizing antibodies were titrated by a conventional VNT using cell culture adapted FMDV O1/ Campos on BHK cell monolayers as described in the OIE Manual of Diagnostic Tests and Vaccines for Terrestrial Animals (OIE, 2012) and adapted by Bucafusco et al. (2014). The endpoint titers of the serum samples were expressed as the logarithm $\left(\log _{10}\right)$ of the reciprocal of the last dilution of serum that neutralized 100 $\times$ tissue culture infective doses $50 \%\left(\right.$ TCID $\left._{50}\right)$ of the virus in $50 \%$ of the wells. To avoid variability between tests, FMDV-neutralizing antibodies were all assessed in one test run.

Total anti-FMDV O1/Campos antibody responses were assessed by LPBE performed as described in the OIE Manual (OIE, 2012), using a rabbit antiserum to capture the virus from an inactivated O1/Campos preparation from clarified infected cell cultures, and a guinea pig anti-FMDV O1/Campos hyperimmune sera as detector antibody (Bucafusco et al., 2014). Serum samples were incubated with a standardized dilution of inactivated virus, and free (unbound) virus was revealed using the detector antibody preparation. Antibody titers were expressed as the reciprocal $\log _{10}$ of serum dilutions giving $50 \%$ of the absorbance recorded in the virus control wells without serum.

Immunoglobulin $\mathrm{G}$ isotype profiles of FMDV-specific bovine antibodies were determined by an indirect ELISA according to Lavoria et al. (2012), using purified inactivated whole FMDV capsids as capture antigen and sheep anti-bovine $\operatorname{IgG}_{1}$ and $\operatorname{IgG}_{2}$ horseradish peroxidase (HRP)-conjugated antibodies diluted 1:750 as detectors (BD-Serotec, Oxford, UK). Immunoglobulin $\mathrm{M}$ determinations were run according to Bucafusco et al. (2014), using sheep anti-bovine IgM HRP-conjugated antibody diluted 1:5,000 as detector (BD-Serotec). Isotype antibody titers were expressed as the highest dilution of the serum reaching an optical density (OD) value equal to mean OD obtained from 25 negative sera \pm 2 SD.

\section{Interferon- $Y$ Assay}

Heparinized whole blood was incubated at $37^{\circ} \mathrm{C}$ for $24 \mathrm{~h}$ with pokeweed mitogen $(10 \mu \mathrm{g} / \mathrm{mL})$ as the positive control, purified inactivated FMDV 140S particles of each vaccine strain (A24/Cruzeiro, A/Arg/2001, O1/ Campos, and C3/Indaial), or PBS as the negative control. Stimulated plasma samples were tested for IFN- $\gamma$ using a commercial kit (Bovigam assay, Thermo Fisher Scientific, Waltham, MA) in triplicate, as described previously (Bucafusco et al., 2015).

\section{Lymphocyte Proliferation Assay}

Peripheral blood mononuclear cells (PBMC) were purified using Histopaque 1083 (Sigma-Aldrich, St. Louis, MO) at 1,000 $\times g$ for $30 \mathrm{~min}$. Resulting PBMC were stained with CellTrace CFSE Cell Proliferation Kit (Life Technologies, Carlsbad, CA) according to manufacturer's instructions and following previously standardized procedures (Mansilla et al., 2013) with some modifications. Briefly, carboxyfluorescein succinimidyl ester (CFSE)-stained PBMC $\left(5 \times 10^{5}\right.$ cells/ well; 96-well plates) from each individual animal were placed in RPMI medium with $10 \%$ calf fetal serum and stimulated, in triplicate. The PBMC were then treated with $5 \mu \mathrm{g} / \mathrm{mL}$ of concanavalin A (ConA, SigmaAldrich), purified inactivated FMDV 140S particles of each vaccine strain, or RPMI as the negative control for $5 \mathrm{~d}$ at $37^{\circ} \mathrm{C}, 5 \% \mathrm{CO}_{2}$. The intensity of CFSE fluorescence was analyzed on a flow cytometer at $530 \mathrm{~nm}$ (BD Biosciences FACSCalibur flow cytometer; BD CellQuest Pro Software) and cell proliferation in each well was estimated as the reduction in CFSE signal of each cell with respect to mock-stimulated cells and reported as the proliferation index.

\section{Animal Welfare}

Animal experiments were approved by the local animal welfare committee of INTA (CICUAE INTA 42/2013), following international animal welfare guidelines. Management and use of colostrum-deprived calves as an animal model followed institutional guidelines (Malacari, 2016).

\section{Data Analysis}

Individual curves were built for all serological assays using serum samples from 5 animals fed 2 L of colostrum each. Each curve was subjected to a 1-phase decay nonlinear regression analysis from which a rate constant $(\mathbf{K})$ was calculated (Table 1). From these val- 
ues, an average $\mathrm{K}$ was computed for each serological assay to estimate the half-life of the MAB using the following formula: average half-life (days) $=\ln (2) / \mathrm{K}$.

The expected protection percentage (EPP) was used to estimate and quantify protective vaccine-induced responses. The EPP represents the correlation between antibody titers at $60 \mathrm{DPV}$, measured by LPBE or VNT, and the percentages of protection achieved for the same groups of animals after in vivo challenge experiments, performed at $90 \mathrm{DPV}$ based on the test for protection against generalized foot infection (Casas Olascoaga et al., 1991; Maradei et al., 2008; OIE, 2012). Titers corresponding to EPP values $\geq 75 \%$ (EPP $\mathbf{7 5 \%}$ ) for the FMDV O1/Campos strain were 1.66 for VNT (Casas Olascoaga et al., 1991) and 2.11 for LPBE (Maradei et al., 2008). Expected MAB decay curves were calculated for each animal, with the antibody titers registered 18 $\mathrm{h}$ after colostrum intake representing the initial titer and the corresponding $\mathrm{K}$ value being applied for each serological parameter. Mean antibody titers observed for each experimental group were pair-wise compared at each time point to those of the expected MAB decay curve using the Mann-Whitney test.

Results obtained for cell-mediated immunity (CMI) tests for each individual against the different FMDV strains performed at each time point were compared using 1-way ANOVA Kruskal-Wallis test, followed by Dunn's test. Analysis of the kinetics of T-cell responses considering all virus strains was accomplished by 2 -way ANOVA with repeated measures, followed by Bonferroni multiple comparisons test. Normal distribution of these values was previously confirmed using the D'Agostino-Pearson normality test.

The confidence interval used was $95 \%$ for all determinations. Statistical analyses were carried out using GraphPad Prism v5.0 (GraphPad Software, La Jolla, CA).

\section{RESULTS}

\section{Passive Transfer of Anti-FMDV-01/Campos Antibodies Through Colostrum}

Titers for neutralizing and total antibodies, as well as for $\operatorname{IgM}, \operatorname{IgG}_{1}$, and $\operatorname{IgG}_{2}$ against FMDV O1/Campos, were initially determined in all colostrum preparations. Pooled AC presented slightly higher mean titers than WC for VNT, resulting marginally below for the remaining serological determinations (Figure 1A). To test the efficacy of the passive immunization procedures, calves fed with the $\mathrm{AC}$ or WC preparations were bled $1 \mathrm{~d}$ post colostrum intake and serum samples were assayed for FMDV-specific antibodies. As shown in Figure 1B, all colostrum-fed animals passively acquired anti-FMDV
Table 1. Average half-life of antibodies (in days) in colostrum preparations measured by the different serological assays ${ }^{1}$

\begin{tabular}{lccc}
\hline Assay $^{2}$ & $\mathrm{~K}(\mathrm{SD})$ & $\mathrm{R}^{2}(\mathrm{SD})$ & $\begin{array}{c}\text { Estimated } \\
\text { half-life }(\mathrm{SD})\end{array}$ \\
\hline $\mathrm{LPBE}$ & $0.042(0.011)$ & $0.98(0.011)$ & $17.85(5.6)$ \\
$\mathrm{VNT}$ & $0.041(0.015)$ & $0.98(0.021)$ & $18.12(5.9)$ \\
$\mathrm{IgG}_{1}$ & $0.044(0.013)$ & $0.99(0.006)$ & $16.56(5.6)$ \\
$\mathrm{IgG}_{2}$ & $0.038(0.014)$ & $0.99(0.006)$ & $20.43(6.4)$ \\
IgM & $0.046(0.007)$ & $0.99(0.005)$ & $15.35(2.5)$ \\
\hline
\end{tabular}

${ }^{1} \mathrm{~K}$ (rate constant) and $\mathrm{R}^{2}$ represent average values obtained from nonlinear regressions built for each individual $(\mathrm{n}=6)$.

${ }^{2} \mathrm{LPBE}=$ liquid phase blocking ELISA; VNT = virus neutralization test.

O1/Campos antibodies as detected by the different serological tests. For all the determinations, antibody titers in colostrum-fed animals were significantly higher than those of CD calves $(P<0.05)$. We did not find any differences between $\mathrm{AC}$ and $\mathrm{WC}$ groups for serological determinations $(P=0.571)$, except for $\operatorname{Ig} G_{2}$ for which the mean antibody titers were significantly higher in the WC group than in the AC calves $(P=0.032)$.

\section{Kinetics of Passively Acquired Antibody Titers}

To evaluate the induction of humoral responses after vaccination in the different experimental groups, we first determined the natural decay of the transferred antibodies. Individual kinetics of extinction were calculated considering the antibody titers at $32 \mathrm{~d}$ of age as the initial time point (0 DPV) to determine the average decay slope for different serological tests. The half-life values of FMDV-specific MAB are shown in Table 1, ranging from $15.35 \pm 2.5 \mathrm{~d}$ for IgM to $20.43 \pm 6.4 \mathrm{~d}$ for $\mathrm{IgG}_{2}$. The half-lives of $\operatorname{IgG}_{1}$ and total and neutralizing antibodies were similar, with mean values between 16 to $18 \mathrm{~d}$.

\section{Progression of Total and Neutralizing Antibody Responses Induced by Vaccination}

The progression of antibody responses after vaccination was assessed by measuring the kinetics of total, neutralizing, and isotype antibody responses against FMDV O1/Campos in sequential serum samples. Curves for total antibodies obtained in calves fed with AC (Figure 2A, LPBE) displayed a slight increase at 28 DPV for some animals, although these values did not differ from those expected in the absence of vaccination (MAB expected decay curves). Similarly, none of the calves in the WC group showed an ascendant trend in total antibody titers after vaccination. In both experimental groups, titers remained above the EPP $75 \%$ level up to the end of the study and kinetic curves were 
A

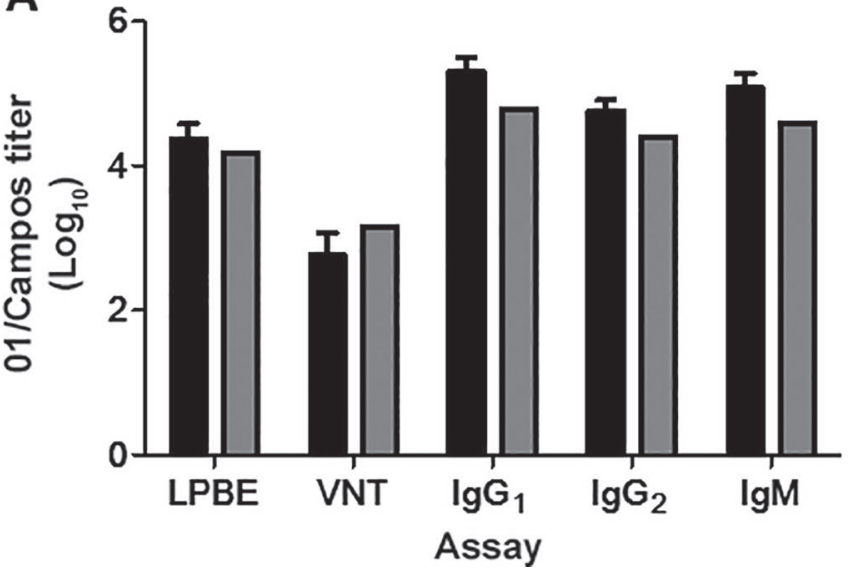

B

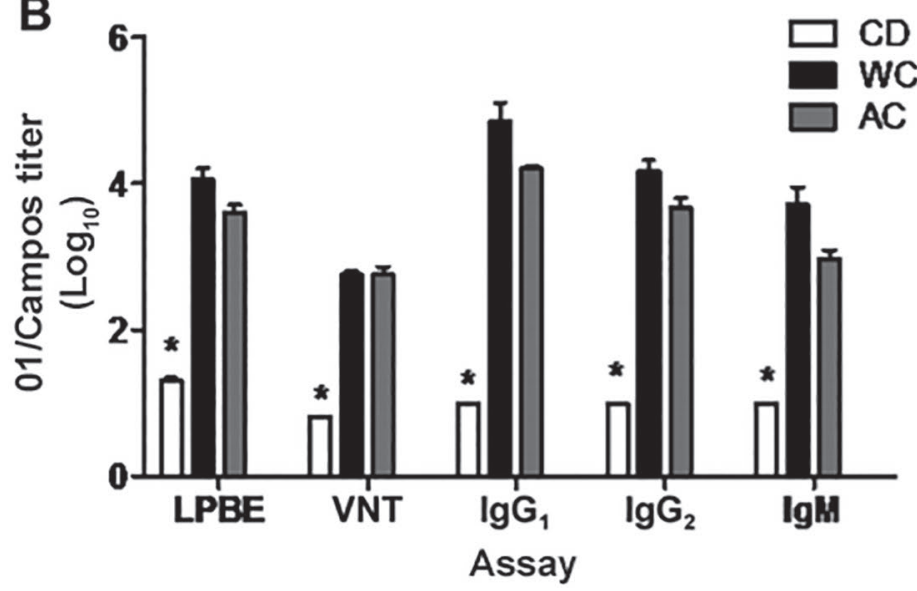

Figure 1. Antibody titers against foot-and-mouth disease virus (FMDV) O1/Campos measured by different serological assays in colostrum preparations (A) and in calves (B) $18 \mathrm{~h}$ after colostrum intake. (A) Titers for acellular colostrum (AC) correspond to the pooled colostrum preparation, and for the whole colostrum (WC) preparation, the titers represent mean values of individual samples \pm SD (n $=3$ ). (B) Mean values $\pm \mathrm{SD}(\mathrm{n}=3)$ in each serological test for all experimental groups; $\mathrm{CD}=$ colostrum deprived: ${ }^{*} P<0.05$ compared with colostrum-fed animals. LPBE = liquid phase blocking ELISA; VNT = virus neutralization test.

identical to those from the decay curves expected in the absence of vaccination. Interestingly, titers at the time of vaccination in this group were higher than those of $\mathrm{AC}$, although these differences were not statistically significant $(P=0.08)$. In contrast, total antibody titers against FMDV O1/Campos were first detected at 14 DPV in CD calves and surpassed the EPP $75 \%$ only at 28 DPV (Figure 2A).

Coinciding with the LPBE results, no significant differences were found in the kinetics of VNT titers measured in colostrum-fed calves $(P>0.2$ at each time point), and titers were similar to those expected over time following the natural waning of MAB. Some calves that received $\mathrm{AC}$ had a slight rise in VNT titers, although it did not significantly differ from the predicted MAB decay slope $(P>0.57)$. Animals deprived of colostrum responded to vaccination with an increase in VNT titers, but the average titers did not reach the EPP $75 \%$ threshold during the experimental period (Figure 2B).

\section{Progression of Isotype Antibody Responses Induced by Vaccination}

Vaccination in both groups of colostrum-fed calves did not elicit significant changes in the isotype profile and kinetics against FMDV O1/Campos strain (Figures $2 \mathrm{C}$ and $\mathrm{D}$ ) compared with those expected in the absence of vaccination (estimated from decay curves). Colostrum-deprived calves developed a typical primary response after vaccination, characterized by a sustained increase in IgM from 7 and up to 14 DPV (Figure 2E), which decreased thereafter, followed by a switch toward
IgG. Immunoglobulin $\mathrm{G}_{1}$ titers peaked at 28 DPV (Figure $2 \mathrm{C}$ ), whereas $\operatorname{IgG}_{2}$ antibodies were first detected between 21 and $28 \mathrm{DPV}$, presenting lower magnitude and a more delayed progression than the $\operatorname{IgG}_{1}$ isotype (Figure 2D).

\section{Cell-Mediated Immunity}

Adaptive cellular responses elicited after vaccination were determined by assessing antigen-specific IFN- $\gamma$ production and lymphoproliferation in whole blood and PBMC samples, respectively, obtained at different times post vaccination. For both CMI tests, no significant differences were found in the mean values obtained after stimulation with the different FMDV vaccine strains for each individual animal at each time point $(P$ $>0.05,1$-way ANOVA). Based on these results, and to analyze the kinetics of T-cell responses and allow comparisons among groups, mean values obtained against the different strains were taken as replicates $(\mathrm{n}=4$ per animal and per time). These values were normally distributed and were analyzed by 2-way ANOVA with repeated measures followed by the Bonferroni multiple comparisons test.

Production of FMDV-specific IFN- $\gamma$ before vaccination was only observed in the AC group, although with high variability among samples. As expected, FMDVinduced IFN- $\gamma$ responses increased after vaccination in all experimental groups. Animals receiving $\mathrm{WC}$ and $\mathrm{AC}$ produced IFN- $\gamma$ in response to FMDV antigens already at 7 DPV, and CD animals responded only from 14 DPV. Peak values were observed at $14 \mathrm{DPV}$ in all the groups and IFN- $\gamma$ levels decreased to pre-immune levels 
already at $28 \mathrm{DPV}$ for CD animals (Figure 3A). At all time points and for all the experimental groups, PBS treatment induced very low IFN- $\gamma$ levels $(\leq 200$ $\mathrm{pg} / \mathrm{mL}$ ). Additionally, all stimulated cells were viable and metabolically active as shown by their response to pokeweed mitogen at concentrations over $3,000 \mathrm{pg} / \mathrm{mL}$ (data not shown).

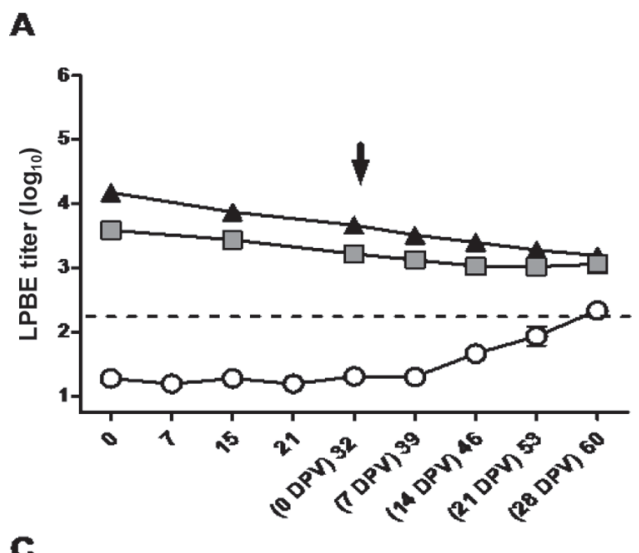

C

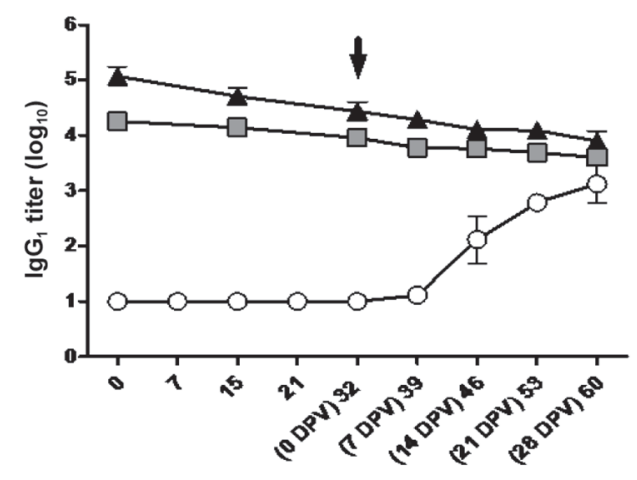

E

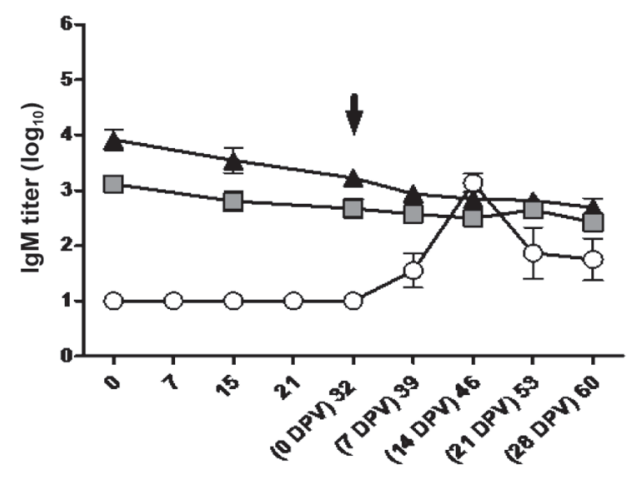

Peripheral blood mononuclear cells were purified from whole blood and incubated with ConA, PBS, or FMDV antigen (purified 140S particles from all 4 vaccine strains), and dividing lymphocytes were counted using CFSE as described above. The proliferation rates found in ConA-stimulated PBMC were always above $20 \%$ relative to the rates observed in mock-treated cells.

\section{в}

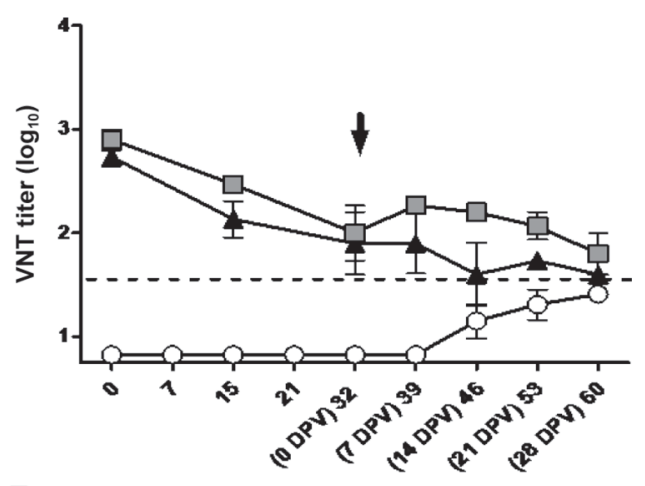

$\mathbf{D}$

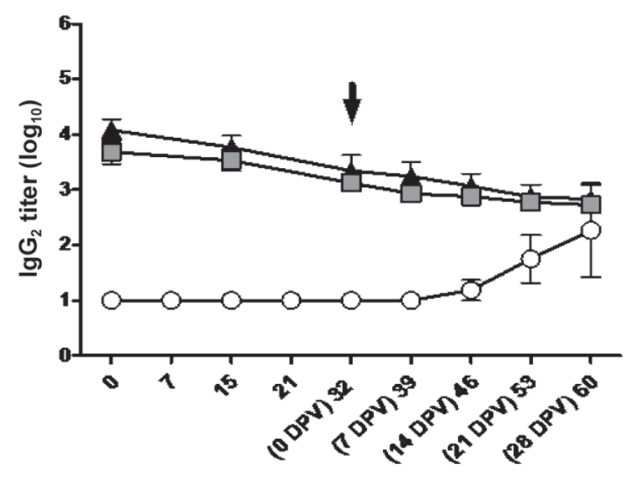

Figure 2. Kinetics of serum antibodies in calves fed with whole colostrum (WC, black triangles) or acellular colostrum (AC, gray squares) or no colostrum (colostrum-deprived, CD, white circles). Blood samples were collected between 0 and $60 \mathrm{~d}$ of age, before and after vaccination, as indicated between parentheses. Antibodies were titered against O1 Campos vaccine strain by liquid-phase blocking ELISA (LPBE; A), the virus neutralization test (VNT; B), and isotype ELISA for $\operatorname{IgG}_{1}(\mathrm{C}), \operatorname{IgG}_{2}(\mathrm{D})$, and $\operatorname{IgM}(\mathrm{E})$. Mean $\log _{10}$ titers \pm SD for each group are depicted. Horizontal dashed lines in panels (A) and (B) indicate the expected protection percentage (EPP) $\geq 75 \%$ for LPBE and VNT, respectively. Arrows indicate the time of vaccination. DPV = days post vaccination. 
Vaccinated animals developed FMDV-specific T-cell responses with similar proliferation rates in response to FMDV antigen stimulation, despite the different colostrum preparations they had received (Figure 3B). The highest proliferation rates for all FMDV strains occurred at $14 \mathrm{DPV}$, except for AC calves, which showed peak responses at $7 \mathrm{DPV}$. T-cell proliferation rates decreased at $28 \mathrm{DPV}$.

Peripheral blood mononuclear cells from WC animals proliferated in response to FMDV antigens before vaccination (at 0 DPV), while prevaccination LPBE values were indistinguishable from the negative (PBS) control responses in the other groups (Figure 3B).

\section{DISCUSSION}

In this study, 2 groups of newborn calves received FMDV-immune colostrum preparations that differed by the presence or absence of maternal cells, with the aim of studying if the presence of maternal immune cells influences the immunity to a commercial FMDinactivated oil-adjuvanted vaccine. Another experimental group not receiving colostrum was also included to identify potential beneficial or detrimental effects of the passive immunity in their capacity to develop adaptive responses after FMD vaccination. Finally, a group of calves fed with a pool of immune colostrums $(\mathrm{n}=5)$

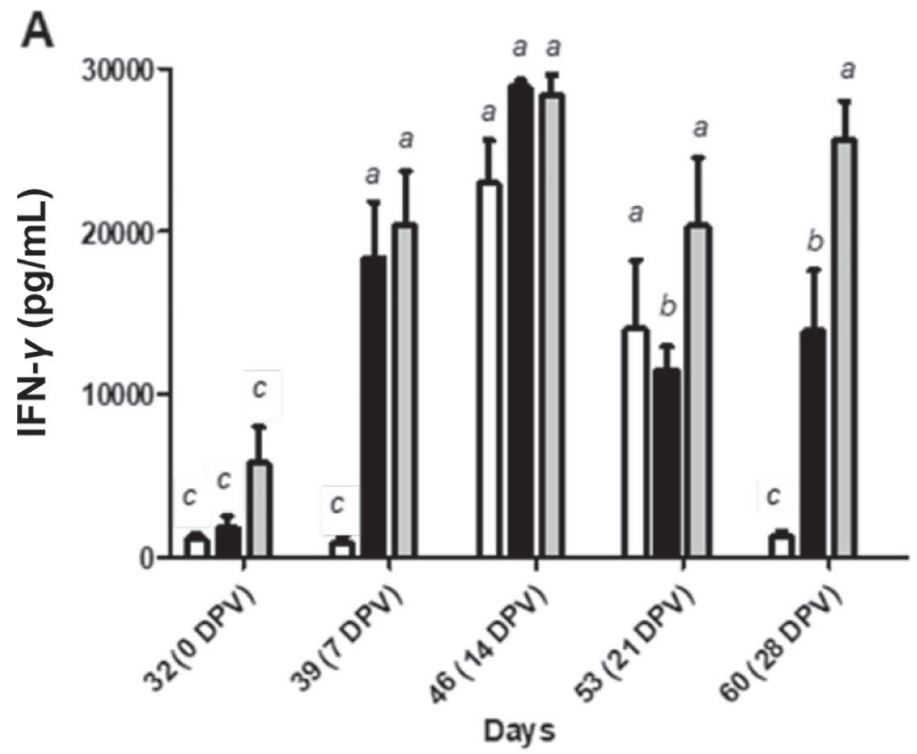

were used to estimate the half-life of MAB based on various serological parameters.

We found slight differences in the FMDV-specific MAB half-life according to the isotype studied. Immunoglobulin $\mathrm{M}$ antibodies had the shortest half-life $(15.35 \pm 2.5 \mathrm{~d})$ and the $\operatorname{IgG}_{2}$ the longest $(20.43 \pm 6.4 \mathrm{~d})$ among the immunoglobulin isotypes. As expected, halflife values obtained for total and FMDV-neutralizing antibodies were between these 2 values and did not differ from one another. As a whole, these results are in agreement with those previously reported by our group (Bucafusco et al., 2014).

Passive transfer of MAB from colostrum was equally efficient in the presence or absence of maternal cells, thus indicating that these immune cells do not have a critical role in the passive acquisition of FMDV-specific antibodies in newborn calves. Moreover, kinetics of antibody waning observed in both experimental groups were practically identical to those estimated through the expected MAB decay curves.

Colostrum-fed animals responded to FMD vaccination with a slight decay in serum antibodies. Interestingly, time-course curves registered after vaccination also followed the same kinetics as those depicting the natural waning of MAB. Only a slight increase was observed in both total (28 DPV) and neutralizing antibodies (7 DPV) in AC calves. This finding may sug-

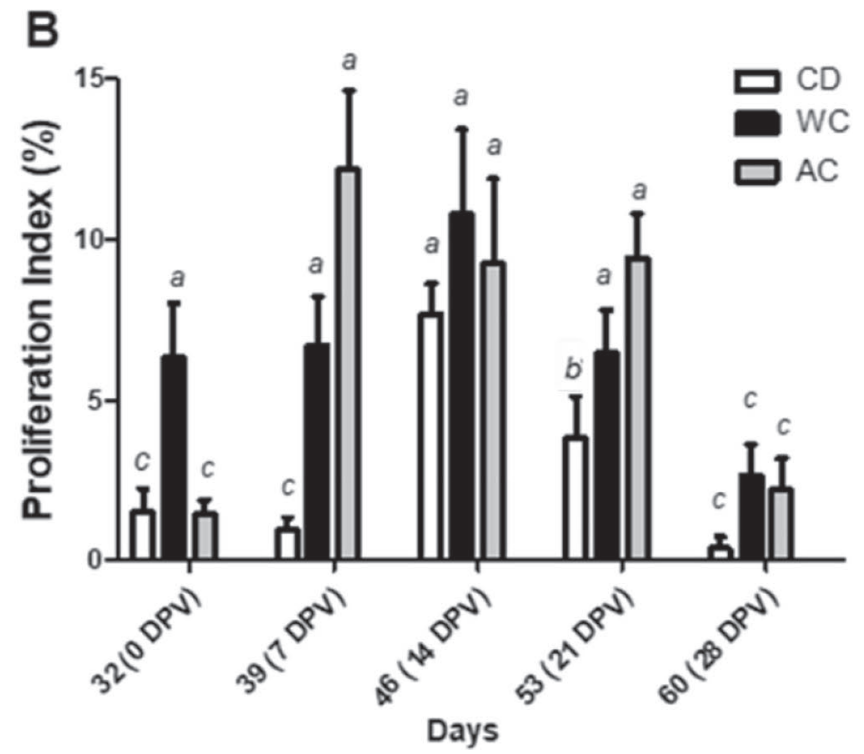

Figure 3. (A) Interferon- $\gamma$ responses measured by ELISA in stimulated plasma after incubating whole blood samples of calves that were colostrum deprived (CD) or fed with whole (WC) or acellular (AC) colostrum with purified 140S particles from the 4-vaccine strain. Values are expressed as the mean optical density $(\mathrm{OD}, 450 \mathrm{~nm}$ ) values $\pm \mathrm{SD}$. (B) Peripheral blood mononuclear cells (PBMC) were purified from whole-blood samples, stained with carboxyfluorescein succinimidyl ester (CFSE) dye and incubated with purified inactivated 140S particles. Proliferation was measured by flow cytometry. Results are depicted as proliferation rate \pm SD with respect to PBS-treated PBMC. Blood samples were taken at different time points before $(0$ days post vaccination, DPV) and after vaccination. Different letters above bars indicate significant differences $(P<0.05)$. 
gest that primary antibody responses cannot be easily detected due to the presence of MAB, even when IgM is titrated. If a primary antibody response was induced in colostrum-fed animals, it might be masked by measurements fitted at the top of a logarithmic scale.

Colostrum-deprived animals developed a primary response after vaccination, evidenced by an increase in IgM titers from $7 \mathrm{DPV}$ (with peak values at $10 \mathrm{DPV}$ ) followed by a switch to $\mathrm{IgG}_{1}$ at $14 \mathrm{DPV}$ and $\mathrm{IgG}_{2}$ a week later. This pattern is the same kinetic described for older animals immunized with an equivalent vaccine from the same manufacturer, as reported previously (Bucafusco et al., 2014); however, total and neutralizing antibody titers were lower than those measured in adult animals (Bucafusco et al., 2014). This result may be due to the immune system still being immature during first month of life. Kampen et al. (2006) reported that the number of $\mathrm{B}$ cells at $30 \mathrm{~d}$ after birth represents only $50 \%$ of the expected number in an adult animal. Other authors attributed a role to the antigens and adjuvants in the generation of antibodies in young cattle (Kampen et al., 2006; Foote et al., 2007). We cannot rule out colostrum components having a role in triggering immune maturation in the newborn individuals.

Some calves that received whole colostrum showed FMDV-specific lymphoproliferation even before vaccination, which may be attributed to the presence of active immune cells passively transferred via colostrum. Unfortunately, the small number of animals included in each group did not enable performing a reliable statistical analysis to confirm the differences observed between WC and CD animals. The transference of maternal cells by colostrum was reported before (Donovan et al., 2007; Reber et al., 2008; Langel et al., 2016). In the study by Donovan et al. (2007), lymphoid cells with proliferative activity were found in colostrum-fed calves vaccinated with bovine viral diarrhea virus, as opposed to the absence of activity in calves that received acellular colostrum. The observed effect had the maximum activity on the first day of life before disappearing completely at $d 7$. Our results indicate that if specific immune cells against FMDV were transferred by colostrum, they might have remained active for at least $40 \mathrm{~d}$ after birth and did not modify the kinetics of $\mathrm{CMI}$ responses over time. Anamnestic IFN- $\gamma$ responses to FMDV were undetectable in $\mathrm{WC}$ calves at $0 \mathrm{DPV}$, suggesting that those FMDV-specific proliferating cells detected before vaccination were not IFN- $\gamma$ producers. After vaccination, FMDV-specific lymphoproliferative responses were found between 7 and $14 \mathrm{DPV}$, with similar magnitude and kinetics in all the animals, regardless of the colostrum preparation they received.
Statistical analysis of the FMDV-specific CMI results showed no differences in the stimulating capacity among the viral strains tested, concurring with a previous report from our group demonstrating that adaptive cellular immunity is highly cross-reactive even among different serotypes (Bucafusco et al., 2015). Interferon- $\gamma$ anamnestic responses peaked at $14 \mathrm{DPV}$ as described before for primary vaccinated adult animals (Bucafusco et al., 2015). Kinetics and magnitude of IFN- $\gamma$ responses were similar in colostrum-fed animals, whether they received immune cells or not. In CD calves, however, IFN- $\gamma$ responses were delayed after vaccination and decreased faster than in colostrum-fed animals. This result can be attributed to the absence immune stimulating factors present in the soluble fraction of colostrum, or even to the absence of specific antibodies that could play a role in facilitating antigen uptake (Robinson et al., 2011). Due to the design of the experiment, we cannot rule out the effect of components of colostrum apart from specific antibodies in shaping the immune response to FMDV vaccine.

In this study, we did not see an evident effect of maternal cells on the kinetics of the immune response induced in vaccinated calves; animals receiving $\mathrm{AC}$ or WC developed similar humoral and cellular FMDVspecific responses. These results are concordant with those reported by Endsley et al. (2003) for bovine viral diarrhea virus infection and counter to findings of Reber et al. (2008), who proposed an active role of maternal cells transferred by colostrum in the activation of calf lymphocytes. Altogether, our results demonstrate that the presence of colostrum maternal cells neither prevent the induction nor represent an advantage for eliciting anti-FMDV CMI after primary vaccination. We did not identify detrimental effects of maternal immunity in the development of vaccine-induced adaptive responses against FMDV. In this scenario, consumption of frozen or whole colostrum in the field will make no difference in the development of immunity against FMDV vaccines. Consequently, regions under mandatory vaccination programs may include young animals.

We conclude that the presence of maternal immune cells transferred from colostrum to the calves do not significantly influence the cellular immune responses to FMDV primary vaccination.

\section{ACKNOWLEDGMENTS}

This work was supported by The National Agency for the Promotion of Science and Technology of Argentina (ANPCyT) though PICT 2011-883 awarded to AVC. $\mathrm{DB}, \mathrm{AVC}$, and MPF are researchers at CONICET. 


\section{REFERENCES}

Belknap, E. B., J. C. Baker, J. S. Patterson, R. D. Walker, D. M. Haines, and E. G. Clark. 1991. The role of passive immunity in bovine respiratory syncytial virus-infected calves. J. Infect. Dis. 163:470-476.

Brito, B. P., A. M. Perez, and A. V. Capozzo. 2014. Accuracy of traditional and novel serology tests for predicting cross-protection in foot-and-mouth disease vaccinated cattle. Vaccine 32:433-436. https://doi.org/10.1016/j.vaccine.2013.12.007.

Bucafusco, D., S. Di Giacomo, J. Pega, M. S. Juncos, J. M. Schammas, M. Pérez-Filgueira, and A. V. Capozzo. 2014. Influence of antibodies transferred by colostrum in the immune responses of calves to current foot-and-mouth disease vaccines. Vaccine 32:6576-6582. https://doi.org/10.1016/j.vaccine.2014.06.056.

Bucafusco, D., S. Di Giacomo, J. Pega, J. M. Schammas, N. Cardoso, A. V. Capozzo, and M. Perez-Filgueira. 2015. Foot-and-mouth disease vaccination induces cross-reactive IFN- $\gamma$ responses in cattle that are dependent on the integrity of the 140S particles. Virology 476:11-18. https://doi.org/10.1016/j.virol.2014.11.023.

Casas Olascoaga, A., V. Astudillo, P. Auge de Melho, A. Young Torres, I. Gomes, M. Kanashiro, Y. Vianna Filho, G. Fernandez, J. R. Baltar, and A. Fernandez. 1991. Correlación de Técnicas de Control de Potencia de la Vacuna Antiaftosa. Proyecto OPS. Cuenca del Plata. Rio de Janeiro, Brasil. 1-10/8/1991.

Donovan, D. C., A. J. Reber, J. D. Gabbard, M. Aceves-Avila, K. L. Galland, K. A. Holbert, L. O. Ely, and D. J. Hurley. 2007. Effect of maternal cells transferred with colostrum on cellular responses to pathogen antigens in neonatal calves. Am. J. Vet. Res. 68:778-782.

Endsley, J. J., J. A. Roth, J. Ridpath, and J. Neill. 2003. Maternal antibody blocks humoral but not $\mathrm{T}$ cell responses to BVDV. Biologicals 31:123-125.

Foote, M. R., B. J. Nonnecke, D. C. Beitz, and W. R. Waters. 2007. Antigen-specific B-cell responses by neonatal calves after early vaccination. J. Dairy Sci. 90:5208-5217. https://doi.org/10.3168/ jds.2007-0285.

Gay, C. C., N. Anderson, N. Fisher, E. W. Fisher, and A. D. McEwan. 1965. Gamma globulin levels and neonatal mortality in market calves. Vet. Rec. 77:148-149.

Goldman, A. S. 1993. The immune system of human milk: antimicrobial, antiinflammatory and immunomodulating properties. Pediatr. Infect. Dis. J. 12:664-671.

Kampen, A. H., I. Olsen, T. Tollersrud, A. K. Storset, and A. Lund. 2006. Lymphocyte subpopulations and neutrophil function in calves during the first 6 months of life. Vet. Immunol. Immunopathol. 113:53-63. https://doi.org/10.1016/j.vetimm.2006.04.001.

Langel, S. N., W. A. Wark, S. N. Garst, R. E. James, M. L. McGilliard, C. S. Petersson-Wolfe, and I. Kanevsky-Mullarky. 2015. Effect of feeding whole compared with cell-free colostrum on calf immune status: The neonatal period. J. Dairy Sci. 98:3729-3740. https://doi.org/10.3168/jds.2014-8422.

Langel, S. N., W. A. Wark, S. N. Garst, R. E. James, M. L. McGilliard, C. S. Petersson-Wolfe, and I. Kanevsky-Mullarky. 2016. Effect of feeding whole compared with cell-free colostrum on calf immune status: Vaccination response. J. Dairy Sci. 99:3979-3994. https://doi.org/10.3168/jds.2015-9892.
Lavoria, M. Á., S. Di-Giacomo, D. Bucafusco, O. L. Franco-Mahecha, D. M. Pérez-Filgueira, and A. V. Capozzo. 2012. Avidity and subtyping of specific antibodies applied to the indirect assessment of heterologous protection against foot-and-mouth disease virus in cattle. Vaccine 30:6845-6850. https://doi.org/10.1016/j.vaccine .2012.09.011.

Malacari, D. A. 2016. Guía para la crianza y mantenimiento del ternero privado de calostro para su utilización como modelo animal. E. INTA, ed. Instituto Nacional de Tecnología Agropecuaria, Buenos Aires, Argentina.

Malacari, D. A., A. Pécora, M. S. Pérez Aguirreburualde, N. P. Cardoso, A. C. Odeón, and A. V. Capozzo. 2018. In vitro and in vivo characterization of a typical and a high pathogenic bovine viral diarrhea virus type II strains. Front Vet Sci. 5:75. https://doi.org/ 10.3389 /fvets.2018.00075.

Mansilla, F. C. C., W. Czepluch, D. A. A. Malacari, Y. P. P. Hecker, D. Bucafusco, O. L. L. Franco-Mahecha, D. P. P. Moore, and A. V. V. Capozzo. 2013. Dose-dependent immunogenicity of a soluble Neospora caninum tachyzoite-extract vaccine formulated with a soy lecithin/beta-glucan adjuvant in cattle. Vet. Parasitol. 197. https://doi.org/10.1016/j.vetpar.2013.04.040.

Maradei, E., J. La Torre, B. Robiolo, J. Esteves, C. Seki, A. Pedemonte, M. Iglesias, R. D'Aloia, and N. Mattion. 2008. Updating of the correlation between lpELISA titers and protection from virus challenge for the assessment of the potency of polyvalent aphtovirus vaccines in Argentina. Vaccine 26:6577-6586. https://doi.org/ 10.1016/j.vaccine.2008.09.033.

Mohr, J. A., R. Leu, and W. Mabry. 1970. Colostral leukocytes. J. Surg. Oncol. 2:163-167.

OIE. 2012. Foot-and-Mouth Disease. Manual of Diagnostic Tests and Vaccines for Terrestrial Animals (Mammals, Birds and Bees). OIE Paris, France.

Reber, A. J., D. C. Donovan, J. Gabbard, K. Galland, M. AcevesAvila, K. A. Holbert, L. Marshall, and D. J. Hurley. 2008. Transfer of maternal colostral leukocytes promotes development of the neonatal immune system Part II. Effects on neonatal lymphocytes. Vet. Immunol. Immunopathol. 123:305-313.

Ridpath, J. E., J. D. Neill, J. Endsley, and J. A. Roth. 2003. Effect of passive immunity on the development of a protective immune response against bovine viral diarrhea virus in calves. Am. J. Vet. Res. 64:65-69.

Robinson, L., M. Windsor, K. McLaughlin, J. Hope, T. Jackson, and B. Charleston. 2011. Foot-and-mouth disease virus exhibits an altered tropism in the presence of specific immunoglobulins, enabling productive infection and killing of dendritic cells. J. Virol 85:2212-2223. https://doi.org/10.1128/JVI.02180-10.

SENASA. 2006. Resolución 351/2006. Servicio Nacional de Sanidad y Calidad Agroalimentaria, Buenos Aires, Argentina.

SENASA. 2013. Resolución 82/2013. Servicio Nacional de Sanidad y Calidad Agroalimentaria Resolución, Buenos Aires, Argentina.

Vitour, D., J. Guillotin, C. Sailleau, C. Viarouge, A. Desprat, F. Wolff, G. Belbis, B. Durand, L. Bakkali-Kassimi, E. Breard, S. Zientara, and G. Zanella. 2011. Colostral antibody induced interference of inactivated bluetongue serotype- 8 vaccines in calves. Vet. Res. 42:18. https://doi.org/10.1186/1297-9716-42-18. 